\title{
Hiperodoncia múltiple y su relación con la displasia cleidocraneal
}

\section{Multiple hyperdontia and it's relationship with cleidocranial dysplasia}

\author{
Harris Ricardo J*, Rebolledo Cobos M**, Natalia Fortich Mesa N***
}

\section{RESUMEN}

La hiperodoncia son alteraciones del desarrollo dentario que exceden de la fórmula dental normal, se presentan diversas clasificaciones dependiendo de su localización, forma, número y medio ambiente, la hiperactividad de las células epiteliales de la lámina dental y la dicotomía son las teorías más aceptadas que intentar explicar su etiología; frecuentemente se detectan en el examen radiográfico de rutina. El diagnóstico y tratamiento temprano de un diente supernumerario, evita una serie de complicaciones como retrasos en su erupción dental, rotaciones, desplazamientos, apiñamiento, diastemas, entre otros; también pueden estar asociados a síndromes genéticos como la Displasia cleidocraneal.

Se reporta caso de paciente masculino de 12 años, que presenta características clínicas y radiográficas compatibles con la Displasia cleidocraneal, en el cual se observaron 8 dientes supernumerarios, 4 localizados en el maxilar y 4 en la mandíbula.

Palabras clave: Diente supernumerario, displasia cleidocraneal, maloclusión.

\section{SUMMARY}

The hiperodoncia are disorders of tooth development that exceed the normal dental formula, there are different classifications depending on their location, shape, number and environment, the hyperactivity of the epithelial cells of the dental lamina and the dichotomy are the most accepted theories that attempt to explain its etiology, is frequently detected in routine radiographic examination. The diagnosis and early treatment of a supernumerary tooth, avoid a number of complications and delays in tooth eruption, rotation, displacement, crowding, spacing, among others, may also be associated with genetic syndromes such as cleidocranial Dispalsia.

We report the case of male patient 12 years, presenting clinical and radiographic features consistent with cleidocranial Dispalsia, in which supernumerary teeth were 8, 4 located in the jaw and 4 jaw.

Key words: Tooth Supernumerary, cleidocranial dysplasia, malocclusion.

Fecha de recepción: 20 de junio de 2011.

Aceptado para publicación: 20 de julio de 2011.

Harris Ricardo J, Rebolledo Cobos M, Fortich Mesa N. Hiperodoncia múltiple y su relación con la displasia cleidocraneal. Av. Odontoestomatol 2013; 29 (1): 25-29.

* Odontólogo. Universidad del Sinú, Cartagena. Especialista en Estomatología y Cirugía Oral. Universidad de Cartagena. Docente Corporación Universitaria Rafael Núñez.

** Odontóloga. Fundación Universitaria San Martín. Especialista en Estomatología y Cirugía Oral. Universidad de Cartagena. Docente de la Fundación Universitaria San Martín sede Puerto Colombia.

*** Odontóloga. Universidad Javeriana. Especialista en Endodoncia, Universidad de Cartagena. Universidad Nacional. Docente Corporación Universitaria Rafael Núñez. 


\section{INTRODUCCIÓN}

Los dientes supernumerarios o hiperodoncia son anomalías en la dentición en el cual se presenta un aumento en el número normal de órganos dentarios, afectan con mayor frecuencia a las mujeres con una relación de 2:1 y comúnmente se les encuentra localizado en mayor proporción el maxilar que la mandíbula (1).

La etiología es desconocida, sin embargo se exponen dos teorías; la hiperactividad de las células epiteliales de la lámina dental y la escisión o dicotomía del folículo dental. En la primera durante la evolución del germen dentario se presenta una degeneración de la lamina dental quedando remanentes de esta en los maxilares, formando posteriormente el desarrollo de hiperodoncia y en la segunda teoría el folículo se divide en dos partes de las cuales una de éstas corresponde al diente normal y la otra el supernumerario (2).

Para el diagnóstico es muy importante identificar algunos de los signos característicos de los dientes supernumerarios como órganos dentarios retenidos o retrasos en la erupción dental, malposiciones dentarias, apiñamiento, diastemas o cierre prematuro de espacio y formaciones quísticas. Con mayor frecuencia son asintomáticos y por lo general son descubiertos en el examen radiográfico de rutina (3).

Para el estudio imagenológico la elección de la radiografía requerida pasará por la necesidad del clínico, donde identificará la posición, forma y características generales de los dientes, donde se incluyen ortopantomografía, radiografía periapical, oclusal, tomografía computarizada, entre otras (4).

Los dientes supernumerarios se clasifican de varias formas, teniendo en cuenta la posición; mesiodens ubicado entre los incisivos centrales, incisivo lateral el que se localice en zona de incisivos laterales, premolar el cual se localiza en zona de premolares, paramolar que se ubica en zona de molares y distomolar ubicado distal al tercer molar; en los diagnósticos diferenciales se deben incluir patologías como: odontomas, cementoblastoma, osteoma $(5,6)$.

La hiperodoncia puede aparecer de forma espontánea en una generación y estar asociados a síndro- mes genéticos como la Displasia cleidocraneal (DCC), el síndrome de Ehlers Danlos, Down, Fabry Anderson, Gardner. La DCC es una afección displásica esquelética autosómica dominante de alta penetrancia, que afecta el desarrollo óseo con alteración de la osificación membranosa y endocondral, causada por mutaciones heterocigotas en el gen CBFA1/RUNX2 (7); clínicamente se caracterizan por ser de baja estatura, braquiocefálicos, hueso frontal prominente, con hipertelorismo, retraso en el cierre de las suturas craneales, hombros caídos, fosas claviculares poco desarrolladas, tórax de forma acampanado, es común aproximar los hombros hacia la línea media anterior del tórax y longitud asimétrica de los dedos, a nivel bucal presentan paladar ojival, maloclusión dental, prognatismo mandibular, dientes supernumerarios, retraso en la erupción de los dientes permanentes, apiñamiento dentario $(8,9)$.

Radiográficamente es habitual que se muestre la formación incompleta o ausencia de clavículas, escápulas hipoplásicas, retraso en la osificación del hueso pélvico, sínfisis del pubis amplia, a nivel bucal se observan múltiples dientes supernumerarios impactados que son característicos de la patología, ausencia del cemento radicular y pueden presentar hipoplasia maxilar $(10,11)$.

La terapéutica indicada para la hiperodoncia es quirúrgica complementando en algunos casos con aparatología ortopédica u ortodóntica, por esta razón el tratamiento debe ser multidisciplinario donde se integren diferentes campos como cirugía oral, ortopedia maxilar, ortodoncia y odontopediatría, para lograr una correcta planificación y manejo de la alteración, la terapia quirúrgica de los supernumerarios es importante porque estos se convierten en un obstáculo para la erupción de los dientes permanentes, alteran la alineación de los dientes en la arcada o pueden estar afectando la estética (12-14).

\section{CASO CLÍNICO}

Paciente masculino 12 años de edad remitido al servicio de Estomatología y Cirugía Oral de la Corporación Universitaria Rafael Núñez por presentar retraso 
en la cronología de erupción de la dentición permanente. En la valoración por el servicio los antecedentes familiares no son relevantes para el caso, en los antecedentes personales la madre manifestó que el paciente presentó un cuadro clínico con neumonía, taquicardia y cefaleas frecuentes hace 5 años.

En el examen físico general presentó facie en forma de gota invertida, hipotelorismo, hombros caídos, tórax estrecho, fosas claviculares poco desarrolladas, gran movilidad de los hombros al aproximarlos hacia la línea media anterior del tórax, un peso de 31 Kg y 1,26 m de talla (Figura 1).

Al examen intrabucal presentó paladar alto, dentición mixta tardía, supernumerario en zona anterior maxilar, pseudoanodoncia del diente número 11 , apiñamiento dental en zona anteroinferior, rotación del diente 42, opacidad café en superficie húmeda en los dientes 65, 26, 74, 75, 36, 84, 85 y 46.

En el estudio imagenológico se observó en la radiografía de tórax, hipoplasia clavicular y tórax en forma

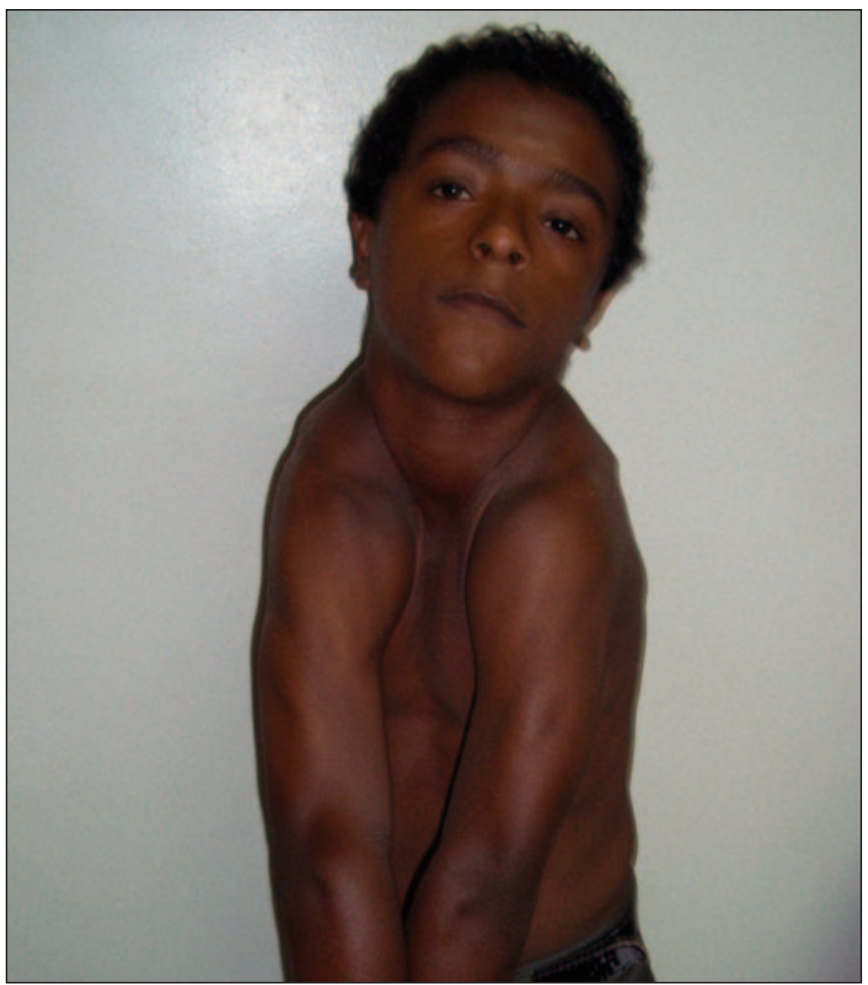

Fig. 1. Hiperfuncionalidad de la articulación del hombro al aproximarlos hacia línea media anterior del tórax.

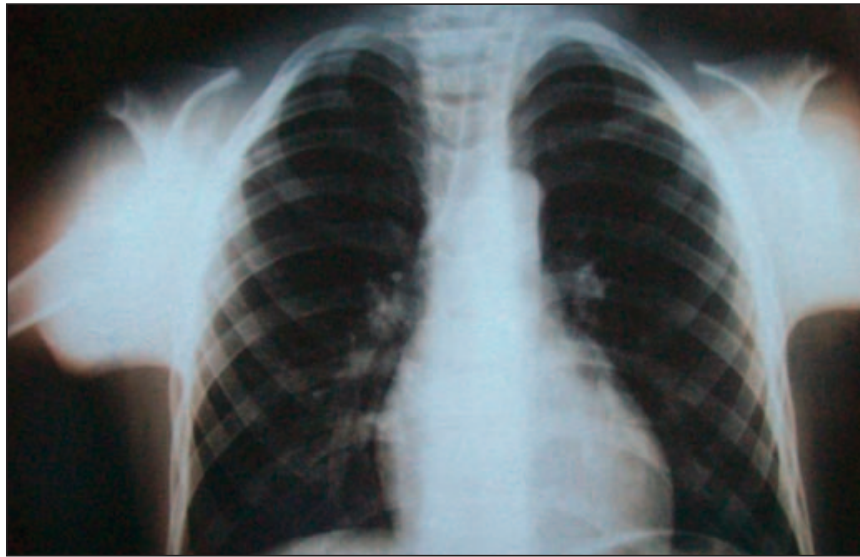

Fig. 2. Radiografía de tórax donde se observa hipoplasia clavicular y tórax en forma de campana.

de campana (Figura 2), en la ortopantomografía se mostró la presencia de ocho supernumerarios siete incluidos y uno erupcionado, cuatro localizados en zona anterior del maxilar y cuatro en la mandíbula, dos de estos localizados en área posterior del lado derecho mandibular, mientras que del lado izquierdo mostró, un diente en el área posterior y uno en la anterior (Figura 3), en la tomografía segmentaria anterior se observó la presencia de los cuatro supernumerarios en el maxilar (Figura 4).

En la radiografía lateral de cráneo se realizaron estudios cefalométricos de Steiner y Mc Namara, el primero arrojó un diagnóstico de clasificación esquelética clase II y prognatismo bimaxilar y el segundo un diagnóstico de clasificación esquelética clase II, crecimiento vertical y prognatismo maxilar; parámetros clínicos y radiográficos compatibles con Displasia cleidocraneal.

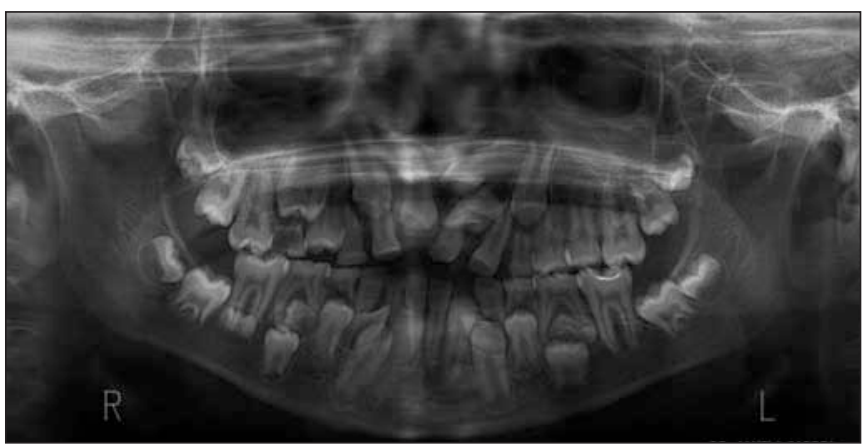

Fig. 3. Ortopantomografía donde se revela la presencia de múltiples supernumerarios. 


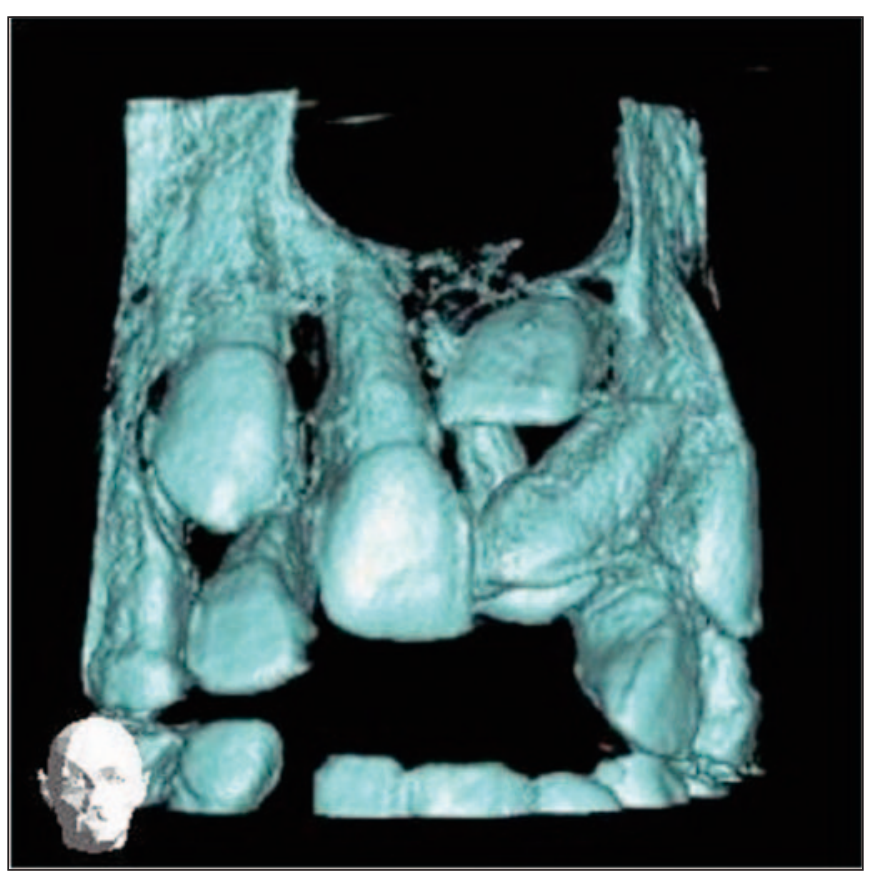

Fig. 4. Tomografía segmentaria donde se muestran supernumerarios en zona anterior del maxilar.

Se traza un plan de tratamiento progresivo a largo plazo en dos fases, la primera constará de profilaxis, enseñanza en técnica de cepillado, restauraciones en resina en los dientes número $65,26,74,75,36$, 84,85 y 46 , seguido del las extracciones de los cuatro dientes supernumerarios localizados en zona anterosuperior, para luego pasar a una segunda fase donde se realizarán las exodoncias de los molares temporales y los cuatro supernumerarios localizados en la mandíbula, continuando con la terapia de ortopedia maxilar donde se colocará un arco lingual que va desde el diente número 36 hasta el 46, para mantener el espacio para la erupción de los dientes 34 , 35,44 y 45 , posteriormente reevaluar el caso para determinar posible tratamiento de ortodoncia.

\section{DISCUSIÓN}

La hiperodoncia o supernumerarios son dientes que exceden de la fórmula dental normal, independientemente de su localización y forma, la etiología es desconocida pero están relacionados en algunos casos a síndromes genéticos como la Displasia cleidocraneal (1).
Gunduz y cols., en el 2007 reportaron que la hiperodoncia es un factor local de tipo dentario que altera el desarrollo oclusal normal y contribuye al establecimiento de maloclusiones originando una serie de complicaciones como apiñamiento dental, rotaciones y desplazamiento de los dientes, retrasos en la cronología de la erupción dentaria (8); concordando con este caso donde el paciente presentó, maloclusión, dentición mixta tardía, mordida abierta, apiñamiento dental en zona anteroinferior y rotación del diente 42 .

Chelvan, en el 2009 y Verstrynge y cols., en el 2006 afirmaron que la DCC es una afección esquelética autosómica dominante, que afecta el desarrollo óseo, originada por mutaciones en el gen CBFA1/RUNX2, entre las manifestaciones clínicas y radiográficas se encuentran la baja estatura, hueso frontal prominente, hipertelorismo, retraso en el cierre de las suturas craneales, hiperfuncionalidad de la articulación del hombro, fosas claviculares poco desarrolladas, maloclusión dental, prognatismo de los maxilares, múltiples dientes supernumerarios, apiñamiento dentario, retraso en la erupción de los dientes permanentes o deciduos, tórax en forma de campana, hipoplasia clavicular $(7,9)$; coincidiendo con las características clínicas y radiográficas de la displasia cleidocraneal encontradas en el presente caso.

Suba y cols., en el 2005, reportaron un caso clínico de paciente con DCC en donde el plan de tratamiento a largo plazo constó de varias etapas, incluyendo fase higiénica, procedimientos quirúrgicos para la extracción de los dientes supernumerarios, fase ortopédica para la expansión transversal del maxilar y la mandíbula, aplicación de una máscara de Delaire para compensar la falta de crecimiento sagital del maxilar y tratamiento protésico (15), en este caso también se trazó un plan de tratamiento progresivo a largo plazo en dos etapas, la primera que constará de fase higiénica, restauraciones en resina en los dientes afectados por caries, exodoncias de los dientes supernumerarios localizados en la zona anterosuperior, la segunda etapa donde se realizaran las exodoncias de los molares temporales y los cuatro supernumerarios localizados en la mandíbula, continuando con la terapia de ortopedia maxilar donde se colocará un arco lingual, para mantener el espacio para la erupción de los dientes 34, 35, 44 y 45, pos- 
teriormente reevaluar el caso para determinar posible tratamiento de ortodoncia.

\section{BIBLIOGRAFÍA}

1. Leco Berrocal MI, Martín Morales JF, Martínez González JM. An observational study of the frequency of supernumerary teeth in a population of 2000 patients. Med Oral Patol Oral Cir Bucal 2007 Mar 1;12(2):134-8.

2. Shah A, Gill DS, Tredwin C, Naini FB. Diagnosis and management of supernumerary teeth. Dent Update 2008 Oct;35(8):510-20.

3. Yagüe-García J, Berini-Aytés L, Gay-Escoda C. Multiple supernumerary teeth not associated with complex syndromes: a retrospective study. Med Oral Patol Oral Cir Bucal 2009 Jul 1;14(7): 331-6.

4. Ferrés-Padró E, Prats-Armengol J, Ferrés-Amat E. A descriptive study of 113 unerupted supernumerary teeth in 79 pediatric patients in Barcelona. Med Oral Patol Oral Cir Bucal 2009 Mar $1 ; 14(3): 146-52$.

5. Arathi R, Ashwini R. Supernumerary teeth: A case report. J Indian Soc Pedod Prev Dent 2005 Jun; 23(2):103-5.

6. Contreras M, Salinas A, Sáez S, Bellet L. Dientes supernumerarios: presentación de dos casos clínicos. Rev Oper Dent Endod 2007;5:60.

7. LiY, Pan W, Xu W, He N, Chen X, Liu H, Darryl Quarles L, Zhou H, Xiao Z. RUNX2 mutations in Chinese patients with cleidocranial dysplasia. Mutagenesis. 2009 Sep;24(5):425-31.

8. Gündüz K, Muglali M. Non-syndrome multiple supernumerary teeth: A case report. J Contemp Dent Pract. 2007 May 1;8(4):81-7.
9. Chelvan HT, Malathi N, Kailasam V, Ponnudurai A. Cleidocranial dysplasia: a family report. J Indian Soc Pedod Prev Dent. 2009 Oct-Dec;27 (4):249-52.

10. Verstrynge A, Carels C, Verdonck A, Mollemans W, Willems G, Schoenaers J. Dentomaxillary and -facial problems in cleidocranial dysplasia. Ned Tijdschr Tandheelkd. 2006 Feb;113(2):69-74.

11. Mata Zubillaga D, Lapeña López de Armentia S. Cleidocranial dysostosis. A review of 11 cases in five generations. An Pediatr (Barc) 2008 Aug;69 (2):162-6.

12. Patchett CL, Crawford PJ, Cameron AC, Stephens $\mathrm{CD}$. The management of supernumerary teeth in childhood - a retrospective study of practice in Bristol Dental Hospital, England and Westmead Dental Hospital, Sydney, Australia. Int J Paediatr Dent. 2001Jul;11(4):259-65.

13. Koch H, Schwarz O, Klausen B. Indications for surgical removal of supernumerary teeth in the premaxilla. Int J Oral Maxillofac Surg 1986 Jun;15 (3):273-81.

14. Olate Morales S, Alister Herdener JP, Thomas Maldonado D, Alveal Campuzano R. Aproximación a un protocolo quirúrgico para el manejo de dientes supernumerarios: Evaluación de un caso y revisión de la literatura. Av. Odontoestomatol 2007;23(2):67-73.

15. Suba Z, Balaton G, Gyulai-Gaál S, Balaton P, Barabás J, Tarján I. Cleidocranial dysplasia: diagnostic criteria and combined treatment. J Craniofac Surg. 2005 Nov;16(6):1122-6.

\section{CORRESPONDENCIA}

Jonathan Harris Ricardo

Correo electrónico: j.harris.r@hotmail.com 